\title{
Lactoferrin in aspirates of odontogenic cyst fluid
}

\author{
A J SMITH, J B MATTHEWS, G I MASON, R M BROWNE \\ From the Department of Oral Pathology, The Dental School, Birmingham
}

SUMMARY The possibility that the presence of lactoferrin in aspirates of odontogenic cyst fluid might be a useful preoperative diagnostic marker for odontogenic keratocyst was investigated. Using qualitative and quantitative immunodiffusion methods fluid from 29 of 29 dental (radicular) cysts, 12 of 14 dentigerous cysts and 27 of 31 keratocysts were found to contain lactoferrin. Although some of the highest concentrations of lactoferrin were detected in fluids from keratocysts, there was no significant difference between lactoferrin concentrations among the three groups. Neutrophil elastase was detected in 20 of 24 samples tested, 22 of which also contained lactoferrin. Immunocytochemical localisation of both lactoferrin and elastase was confined to neutrophils infiltrating cyst walls. These results suggest that lactoferrin in fluid from odontogenic cysts is derived from infiltrating neutrophils and that its presence in aspirated fluids is not a useful diagnostic marker for odontogenic keratocyst.

Because it is more aggressive and tends to recur after surgical excision it is important to differentiate odontogenic keratocyst from other odontogenic cysts. Difficulties in the preoperative diagnosis of odontogenic keratocysts have stimulated attempts to find a biochemical or immunological marker in aspirates of cyst fluid.$^{1-9} \mathrm{~A}$ comparatively simple and widely accepted one is the concentration of total soluble proteins, because fluid from keratocysts contains significantly less soluble protein than fluid from non-keratinising odontogenic cysts. ${ }^{1-3}$ Estimating protein in aspirates of cyst fluid is difficult, however, ${ }^{45}$ and more specific markers for the diagnosis of odontogenic keratocyst have been sought, usually by immunological methods.

Southgate et $a^{6}{ }^{6}$ investigated the keratocyst "prealbumin" band first described by Toller ${ }^{1}$ and associated it with various keratinising epithelia. The keratocyst antigen of Kuusela et al, ${ }^{78}$ which has a neutral isoelectric point, may be related to keratin. Unfortunately these antigens, though they seem to offer a means of specifically identifying keratocysts, are poorly characterised and are dependent on polyclonal antisera raised to whole keratocyst fluid. More recently another keratocyst specific antigen has been reported $^{9}$ (antigen $\left.\mathbf{X}\right)$, which seems to be antigenically similar to lactoferrin. ${ }^{10}$ Because of the potential importance of a marker to which specific antibodies and the purified antigen itself are available, we have sought lactoferrin in fluid aspirates from 74 odontogenic cysts using methods that are available in any laboratory. To parallel these studies, frozen and paraffin wax embedded sections of cyst walls were

Accepted for publication 5 May 1988 stained immunocytochemically for lactoferrin and elastase, both of which are present in neutrophils.

\section{Material and methods}

Fluids were centrifuged at $5000 \mathrm{~g}$ for 10 minutes to separate cellular material and other particulate matter. Fluid aspirates of large enough volume were assayed immediately after centrifugation by the bromolcresol green dye binding method for albumin" and the glyoxylic acid method for globulin. ${ }^{12}$ For all procedures accurate sampling of cyst fluid, which is often mucoid, was carried out using positive displacement pipettes. Specimens of fluid ( 29 from dental (radicular) cysts, 14 from dentigerous cysts, and 31 from keratocysts) were collected over a four year period and stored at $-20^{\circ} \mathrm{C}$ until immunochemical analysis. Diagnosis was based on clinical criteria and histopathological examination of excised tissues.

Surgical specimens were obtained fresh and samples snap frozen and stored in liquid nitrogen. The remainder of each specimen was fixed in neutral formalin and processed to paraffin wax. Twenty five specimens were used for this study and consisted of 10 dental (radicular) cysts, five dentigerous cysts, and 10 odontogenic keratocysts as defined by Browne. ${ }^{13}$

Double immunodiffusion was carried out on 24 fluids in $1.5 \%$ agarose in barbitone buffer (isoelectric point $0.05, \mathrm{pH} 8.6$ ) containing $3 \%$ polyethylene glycol (PEG 6000; Sigma) and allowed to proceed for 48 hours at $4^{\circ} \mathrm{C}$. Quantitation of lactoferrin was carried out by single radial immunodiffusion using purified human lactoferrin (Sigma; isolated from milk) standard, and rabbit antihuman lactoferrin antiserum 
(Dako). Ten $\mathrm{ml}$ agarose $(1.5 \%$ in barbitone buffer plus 3\% PEG) containing $20 \mu \mathrm{l}$ antihuman lactoferrin antibody was used for each $8 \times 8 \mathrm{~cm}$ plate. Diffusion was allowed to proceed for 48 hours at room temperature. Specimens of cyst fluid (64 specimens, $4 \mu \mathrm{l}$ samples) were analysed in duplicate neat and at 1/10 and $1 / 100$ dilutions with duplicate sets of standards on each plate (six dilutions spanning $0.01-0.16 \mathrm{~g} / \mathrm{l}$ lactoferrin). Analysis of data was by the Student's $t$ test.

Immunoperoxidase staining of acetone fixed frozen sections and trypsin treated paraffin wax sections was performed by the peroxidase antiperoxidase (PAP) complex method as previously described. ${ }^{1415}$ Rabbit antihuman lactoferrin, swine antirabbit immunoglobulin, and PAP complexes were obtained from Dako Ltd. Rabbit antihuman neutrophil elastase was prepared in the department of oral pathology using purified elastase as immunogen. Reactivity of this antiserum in immunoperoxidase staining of various human tissues is limited to neutrophils, completely inhibited by purified elastase, and unaffected by preabsorption with human lactoferrin. Both rabbit antisera were used at a dilution of $1 / 500$.

\section{Results}

Quantitation of lactoferrin in fluid from 64 odontogenic cysts by single radial immunodiffusion (table) indicated that detectable concentrations were present in all dental (radicular) samples and most of the dentigerous and keratocyst samples. Though the mean concentration of lactoferrin in keratocyst fluid was higher than that in dental (radicular) or dentigerous fluid, the difference was not significant. By contrast, the mean total soluble protein concentrations in samples of keratocyst fluid were significantly lower than those in dental (radicular) or dentigerous cysts $(p<0.05)$. There was no correlation between concentrations of total soluble protein and lactoferrin $(r=0 \cdot 125)$. Twenty four specimens, including 14 previously examined by single radial immunodiffusion, were investigated for lactoferrin and neutrophil elastase by double immunodiffusion. Both

Table Concentrations of lactoferrin and total soluble protein in aspirates of odontogenic cyst fluid

\begin{tabular}{llll}
\hline & $\begin{array}{l}\text { Dental } \\
\text { (Radicular) }\end{array}$ & Dentigerous & Keratocyst \\
\hline Lactoferrin (g/l) & & & \\
$\quad$ No & 25 & 12 & 27 \\
$\begin{array}{l}\text { Mean (SD) } \\
\text { Range }\end{array}$ & $0 \cdot 145(0 \cdot 133)$ & $0 \cdot 124(0 \cdot 151)$ & $0 \cdot 27(0 \cdot 364)$ \\
$\begin{array}{l}\text { Total protein (g/l) } \\
\text { No }\end{array}$ & $0 \cdot 022-0 \cdot 42$ & $0-0 \cdot 495$ & $0-1 \cdot 515$ \\
Mean (SD) & 18 & 11 & 15 \\
Range & $86 \cdot 2(38 \cdot 1)$ & $87 \cdot 7(32 \cdot 1)$ & $52 \cdot 1(31 \cdot 4)$ \\
\hline
\end{tabular}

components were present in 20 specimens, both absent in two, and lactoferrin alone detected in two. Lines of identity were obtained between the components detected in cyst fluids and purified lactoferrin and elastase. All specimens in which lactoferrin had been detected by single radial immunodiffusion were positive in the double diffusion test. Immunoperoxidase staining for lactoferrin and elastase showed that both components were present within neutrophils infiltrating cyst walls. No other sites of antigen localisation were noted.

\section{Discussion}

This study shows that lactoferrin is present in aspirates of odontogenic cyst fluid irrespective of cyst type. The immunocytochemical evidence that lactoferrin was only detectable in neutrophils, also noted by Douglas and Craig, ${ }^{10}$ and the presence of lactoferrin and elastase together in specimens of fluid, strongly implicates the infiltration of cyst walls by neutrophils as the source of lactoferrin in the samples of fluid. Interestingly, odontogenic keratocysts that are generally considered to be developmental in origin and associated with comparatively small numbers of infiltrating inflammatory cells compared with inflammatory cysts (dental or dentigerous), contained the highest concentrations of lactoferrin. The molecular size of lactoferrin (about 77000 daltons) is about the size that Toller ${ }^{1617}$ suggested was the largest that could diffuse across the epithelial linings of cysts. Our own data, ${ }^{18}$ however, showed that higher molecular weight proteins are also found in fluids from all types of cyst. Epithelial permeability, together with discontinuities in the epithelial lining and intraepithelial channels, ${ }^{19}$ could allow passage of lactoferrin into the cyst luminal fluid. The high concentrations found in fluid from some keratocysts might reflect greater barriers to lactoferrin diffusing out through the lining of the keratocysts, the prolonged half life of lactoferrin in keratocyst fluid, or the lower concentrations of fluid proteins, or both, that may mask the antigenic sites on lactoferrin molecules.

The widespread distribution of lactoferrin in aspirates of odontogenic cyst fluid, and the wide and overlapping ranges of concentrations in fluids from different types of cyst, indicates that it is not a useful marker of odontogenic keratocysts. Furthermore, the identity of the keratocyst specific antigen- $X$ reported by Douglas and Craig' remains to be discovered, and its antigenic relation to lactoferrin requires clarification. It is important, however, that investigations of possible markers of the odontogenic keratocyst based on analysis of its fluid should continue; further characterisation of the keratocyst antigens described by Kuusela et al $^{78}$ and Douglas and Craig may prove worthwhile. In the meantime we consider that the 
combination of protein analysis and detection of epithelial squames ${ }^{45}$ provides the most reliable method for preoperative diagnosis of odontogenic keratocyst using fluid apirates.

We thank Dr D Burnett of the department of immunology for the purified elastase.

\section{References}

1 Toller PA. Protein substances in odontogenic cyst fluids. Br Dent J 1970;128:317-22.

2 Kramer IRH, Toller PA. The use of exfoliative cytology and protein estimations in preoperative diagnosis of odontogenic keratocysts. Int J Oral Surg 1973;2:143-51.

3 Browne RM. Some observations on the fluids of odontogenic cysts. J Oral Pathol 1976;5:74-87.

4 Smith AJ, Smith G, Browne RM. Analysis of odontogenic cyst fluid aspirates. IRCS Med Sci 1986;14:304.

5 Smith AJ, Browne RM. Analysis of odontogenic keratocyst fluids for pre-operative diagnosis. J Clin Pathol 1987;40:354-5.

6 Southgate J, Whicher JT, Davies JD, O'Reilly DStJ, Matthews RW. A protein of squamous keratinising epithelium from odontogenic keratocyst fluid. Virchows Arch (A) 1986;409: 705-13.

7 Kuusela P, Hormia M, Tuompo H, Ylipaavalniemi P. Demonstration and partial characterisation of a novel soluble antigen present in keratocysts. Oncodevelopmental Biology and Medicine 1982;3:283-90.
8 Kuusela P, Ylipaavalniemi P, Thesleff, I. The relationship between the keratocyst antigen (KCA) and keratin. J Oral Pathol 1986;15:287-91.

9 Douglas CWI, Craig GT. Recognition of a protein apparently specific to odontogenic keratocyst fluids. J Clin Pathol 1986;39:1108-15.

19 Douglas CWI, Craig GT. Evidence for the presence of lactoferrin in odontogenic keratocyst fluids. J Clin Pathol 1987;40:914-21.

11 Doumas BT, Watson WA, Briggs HG. Albumin standards and the measurement of serum albumin with bromocresol green. Clin Chim Acta 1971;31:87-96.

12 Goldenberg H, Drewes PA. Direct photometric determination of globulin in serum. Clin Chem 1971;17:358-62.

13 Browne RM. The pathogenesis of odontogenic cysts. A review. J Oral Pathol 1975;4:31-46.

14 Matthews JB, Mason GI. Influence of decalcifying agents on immunoreactivity of formalin-fixed paraffin-embedded tissue. Histochem J 1984;16:771-84.

15 Matthews JB, Browne RM. An immunocytochemical study of the inflmmatory cell infiltrate and epithelial expression of HLA-DR in odontogenic cysts. J Oral Pathol 1987;16:112-7.

16 Toller PA. Permeability of cyst walls in vivo: Investigations with radioactive tracers. Proceedings of the Royal Society of Medicine 1966;59:724-9.

17 Toller PA. The osmolarity of fluids from cysts of the jaws. Br Dent $J$ 1970;129:275-8.

18 Smith G, Smith AJ, Browne RM. Protein differences in odontogenic cyst fluids. IRCS Medical Science 1983;11:117.

19 Cohen MA. Pathways of inflammatory exudate through radicular epithelium. J Oral Pathol 1979;8:369-79.

Requests for reprints to: Dr A J Smith, Department of Oral Pathology, The Dental School, St Chads Queensway, Birmingham B4 6NN. England. 